\title{
Wide field of view common-path lateral-shearing digital holographic interference microscope
}

Priyanka Vora

Vismay Trivedi

Swapnil Mahajan

Nimit Patel

Mugdha Joglekar

Vani Chhaniwal

Ali-Reza Moradi

Bahram Javidi

Arun Anand 


\title{
Wide field of view common-path lateral-shearing digital holographic interference microscope
}

\author{
Priyanka Vora, ${ }^{\mathrm{a}, \mathrm{b}}$ Vismay Trivedi, ${ }^{\mathrm{a}}$ Swapnil Mahajan, ${ }^{\mathrm{a}}$ Nimit Patel, ${ }^{\mathrm{a}}$ Mugdha Joglekar, ${ }^{\mathrm{a}}$ Vani Chhaniwal, ${ }^{\mathrm{a}}$ \\ Ali-Reza Moradi, ${ }^{c, d}$ Bahram Javidi, ${ }^{e}$ and Arun Anand ${ }^{a, *}$ \\ ${ }^{a}$ The Maharaja Sayajirao University of Baroda, Faculty of Technology and Engineering, Department of Applied Physics, Vadodara, Gujarat, India \\ buka Tarsadia University, Department of Physics, Bardoli, Gujarat, India \\ 'Institute for Research in Fundamental Sciences, School of Nano Science, Tehran, Iran \\ dInstitute for Advanced Studies in Basic Sciences, Optics Research Center, Zanjan, Iran \\ eUniversity of Connecticut, Department of Electrical and Computer Engineering, Storrs, Connecticut, United States
}

\begin{abstract}
Quantitative three-dimensional (3-D) imaging of living cells provides important information about the cell morphology and its time variation. Off-axis, digital holographic interference microscopy is an ideal tool for 3-D imaging, parameter extraction, and classification of living cells. Two-beam digital holographic microscopes, which are usually employed, provide high-quality 3-D images of micro-objects, albeit with lower temporal stability. Common-path digital holographic geometries, in which the reference beam is derived from the object beam, provide higher temporal stability along with high-quality 3-D images. Self-referencing geometry is the simplest of the common-path techniques, in which a portion of the object beam itself acts as the reference, leading to compact setups using fewer optical elements. However, it has reduced field of view, and the reference may contain object information. Here, we describe the development of a common-path digital holographic microscope, employing a shearing plate and converting one of the beams into a separate reference by employing a pin-hole. The setup is as compact as self-referencing geometry, while providing field of view as wide as that of a two-beam microscope. The microscope is tested by imaging and quantifying the morphology and dynamics of human erythrocytes. () 2017 Society of Photo-Optical Instrumentation Engineers (SPIE) [DOI: 10.1117/1.JBO.22.12.126001]
\end{abstract}

Keywords: quantitative phase imaging; microscopy; cells; interference; biomedical optics; holography applications.

Paper 170534R received Aug. 16, 2017; accepted for publication Nov. 16, 2017; published online Dec. 12, 2017.

\section{Introduction}

A digital holographic interference microscope (DHIM) in the transmission mode, based on the off-axis superposition of separate object beam with a background reference beam, is an ideal tool for divulging information about the cell morphology and its time variation. ${ }^{1-15}$ This geometry is flexible in the sense that the whole field of view provides useful information and the fringe density can be altered by maneuvering the beam splitting and steering elements. Also, considering the advantage of separating out different diffracted beams in frequency spectrum in off-axis geometry, ${ }^{10,15-18}$ a two-beam setup employing Mach-Zehnder interferometer geometry (MZ-DHIM) is commonly used for the construction of DHIM. ${ }^{1-15}$ Cellular and subcellular structures are observable and quantifiable using this setup, and, from a time series of holograms, time-varying cell parameters under various conditions can also be studied. ${ }^{1-18}$

In the case of MZ-DHIM, two separate coherent beams are used to generate interference patterns. Both beams travel along different paths and may pick up uncorrelated path length variations even when a vibration isolation mechanism is employed. This will lead to reduction in temporal phase stability of the microscope, which in turn may affect its use in the measurement of small cell thickness fluctuations of the order of few tens of nanometer, for example, in the case of human erythrocytes. ${ }^{19,20}$ Moreover, the implementation of a two-beam microscope using MZ-DHIM geometry requires a number of optical components

*Address all correspondence to: Arun Anand, E-mail: aanand-apphy@ msubaroda.ac.in for beam splitting, beam combining, and beam steering, such as mirrors, beam splitters, and in some cases two microscope objectives (MOs), etc., which makes the setup complex, nonportable, and expensive. To overcome the problem of temporal stability, common-path interferometric technique can be used. ${ }^{19-38}$ Common-path techniques duplicate the object wavefront and generate the reference wavefront from one of these duplicated wavefronts. This can be done by either spatially filtering one of the duplicated wavefronts and using it as the reference wavefront ${ }^{19-29}$ or using a portion of the duplicated wavefront (which does not contain object information) as the reference wavefront. $^{30-38}$ A second technique termed a self-referencing DHIM technique requires fewer optical elements and hence leads to very compact off-axis geometries. One of the easiest ways to implement self-referencing geometry is by the use of a glass plate (GP) to create two laterally sheared versions of the object wavefront and then superposing them. . $^{5,35,36}$ Geometry of this setup is simple, easy to implement, and compact, needing only an objective lens and a GP. Two beams follow the same path and encounter the same set of optical elements, reducing unwanted vibrations in the setup. ${ }^{35}$ It has been shown that subnanometer temporal stability over a period of few minutes, which is suitable for studying membrane fluctuations in biological cells, is possible with this geometry. ${ }^{35}$ However, this geometry suffers from the reduction of field of view as only a portion of the field of view (in the case of large detector arrays) contains useful object information. Also, the reference

1083-3668/2017/\$25.00 @ 2017 SPIE 


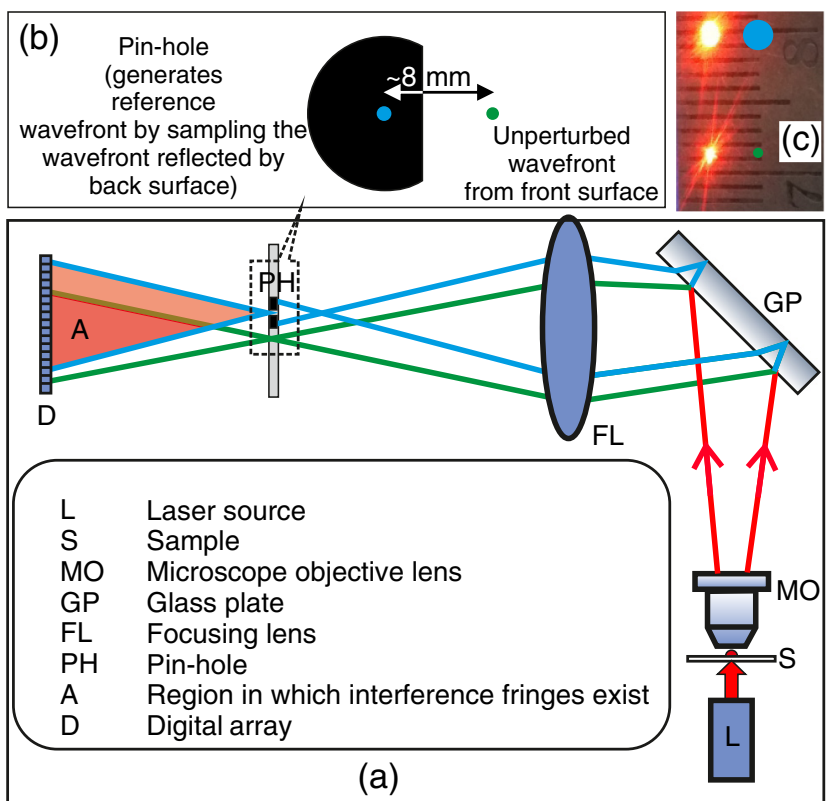

Fig. 1 Wide field of view lateral-shearing DHIM: (a) schematic of the setup, (b) configuration of the pin-hole mounted on a microscope slide used to convert the wavefront reflected from the back surface of the shearing GP into the reference wavefront, and (c) photograph of the object at reference beam at the pin-hole plane. beam, which is the portion of the object beam not modulated by the object, may contain information about the medium surrounding the object, which may result in unwanted spatial phase variations. A setup that is as easy to implement as the self-referencing geometry but provides the three-dimensional (3-D) imaging capability and field of view of MZ-DHIM will have the potential to provide high-quality 3-D images with very high temporal stability. Here, the development of a simple, common-path DHIM by modifying the lateral-shearing digital holographic microscope by the use of a pin-hole to create a separate reference beam, while keeping its common-path nature intact, is described. It is implemented using a laser diode module as the source. An inexpensive version of the microscope is also made using a webcam sensor instead of a CCD array to record holograms. The microscope is tested by 3-D imaging of red blood cells and its thickness fluctuations. Static and dynamic parameters of human red blood cells are also extracted using the same microscope.

\section{Wide Field of View Self-Referencing Digital Holographic Microscope}

Figure 1(a) shows the schematic diagram of a wide field of view lateral-shearing digital holographic microscope. It uses a laser diode module working at a wavelength of $635-\mathrm{nm}$ ( $5 \mathrm{~mW}$, random linearly polarized) as the source. An MO lens $(40 \times$ magnification and NA $=0.65)$ is used for object magnification. The beam from the laser (L) transilluminates the object (S) mounted on a translation stage. The sample is magnified by
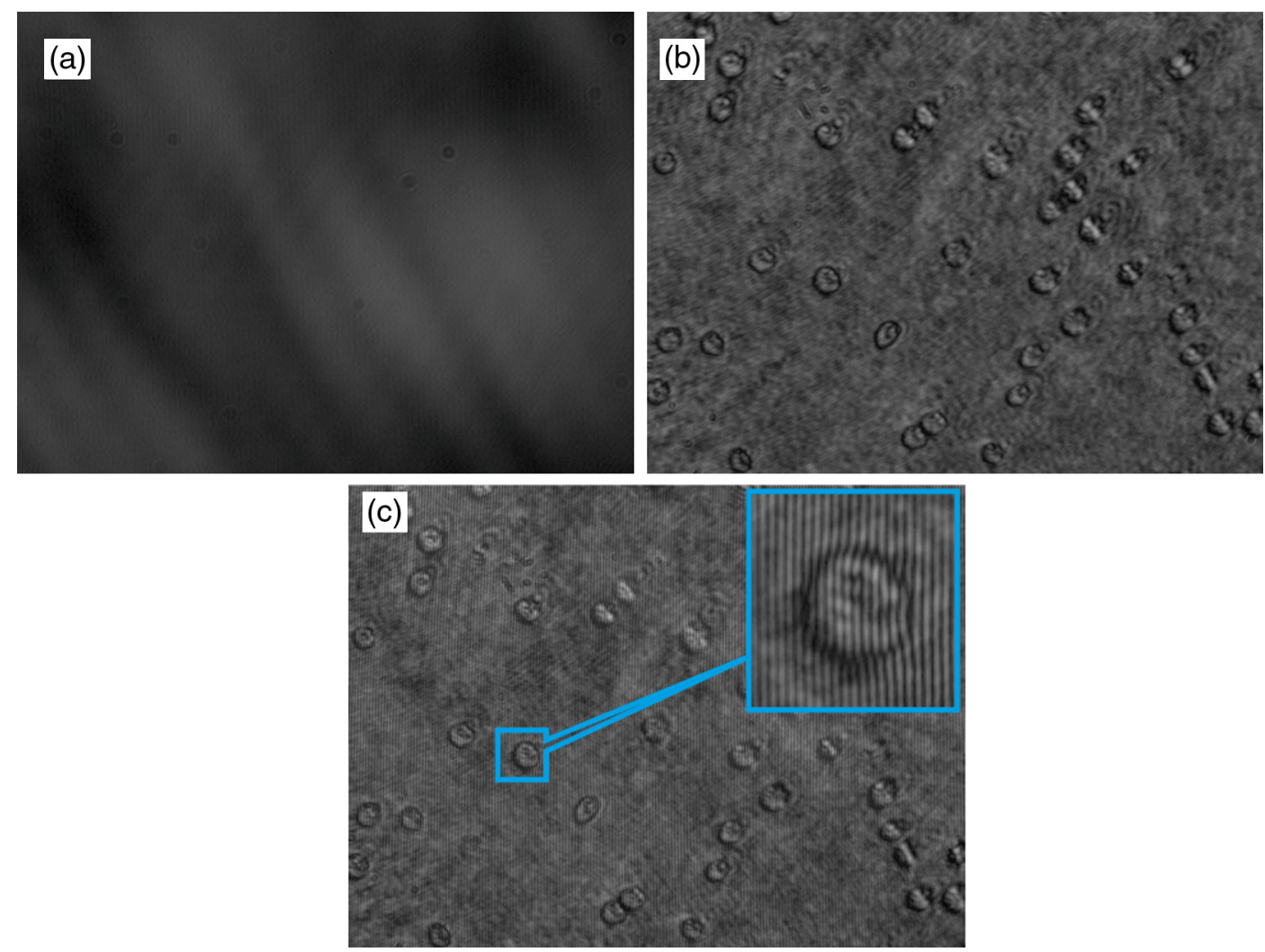

Fig. 2 Recorded images in the wide field of view lateral-shearing digital holographic microscope: (a) reference beam intensity at the detector plane, (b) object beam intensity recorded by the detector, and (c) holograms formed at the detector plane. 
an MO lens. This beam is then split into two laterally sheared versions by the GP made up of fused silica with a thickness of $1 \mathrm{~cm}$. Reflections from the front and back surfaces of the GP travel along the same path but at a slight angle to each other due to shearing introduced by the GP to the spherical wavefront. These two beams are focused by a plano-convex lens of focal length $12.5 \mathrm{~cm}$ having a clear aperture of $5 \mathrm{~cm}$. This lens is kept $25 \mathrm{~cm}$ (MO to front surface of the GP to lens) from the objective lens. The beams from the front and back surface of the GP travel different distances from the GP to reach the focusing lens. The path difference and the lateral separation between the two beams, introduced by the GP, result in the beams coming to focus at different axial as well as lateral positions [Figs. 1(a) and 1(b)]. Assuming that only a very small portion of wavefront incident on the GP is recorded by the digital array D, the path length difference among the wavefronts can be deduced using Snell's law and is

$\delta=n_{g} 2 t \cos \left(\theta_{r}\right)$,

where $n_{g}$ is the refractive index of the shearing GP and $t$ is its thickness. $\theta_{r}$ is the angle of refraction, which depends on the angle of incidence and the refractive indices of the shearing GP as well as the medium surrounding the shearing plate (usually air). For high-contrast interference fringes to form, this path length difference [Eq. (1)] should be less than the coherence length of the laser source. At the pin-hole plane, where the beam from the front surface converges to a point source, the lateral separation between the two beams (from the front surface and the back surface) is $\sim 8 \mathrm{~mm}$. A portion of the wavefront from the back surface is filtered (sampled), using a $30-\mu$ m-diameter pin-hole mounted on a microscope slide [Fig. 1(b)], to erase the object information and is converted into a reference wavefront. ${ }^{25}$ The microscope slide on which the pin-hole is mounted could be translated laterally. The beam from the back surface of the shearing plate is centered on the pin-hole by this lateral translation, to maximize the flux after the pinhole. The beam from the front surface of the GP passes through the unobstructed portion of the microscope slide, without any perturbation, carrying all of the object information. These two beams superimpose at the detector plane (which is also the image plane) resulting in the formation of holograms.

Figures 2(a) and 2(b) show the intensity of the reference and object wavefront at the detector plane, respectively. The hologram formed by their superposition is shown in Fig. 2(c). All of the images shown in Fig. 2 are recorded using an 8-bit CCD array with a $4.65-\mu \mathrm{m}$ pixel pitch. Complex amplitude distribution of the magnified object at the image plane is reconstructed by numerical implementation of the angular spectrum propagation integral $^{39}$
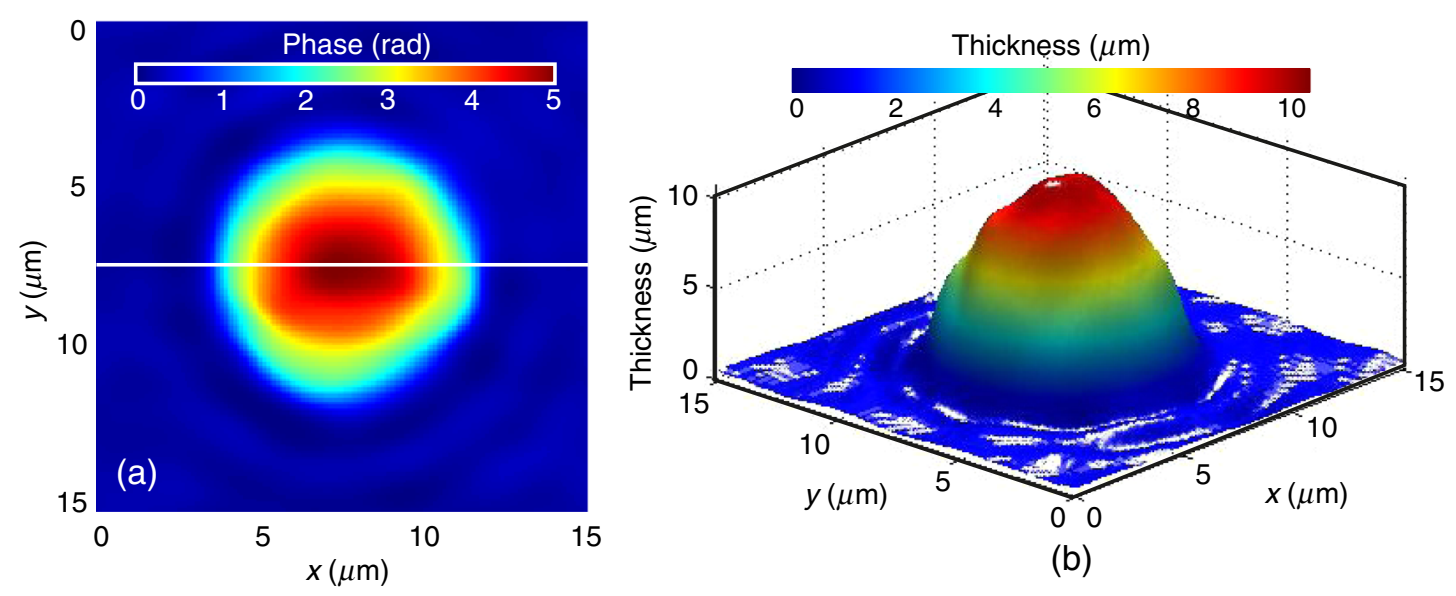

(b)

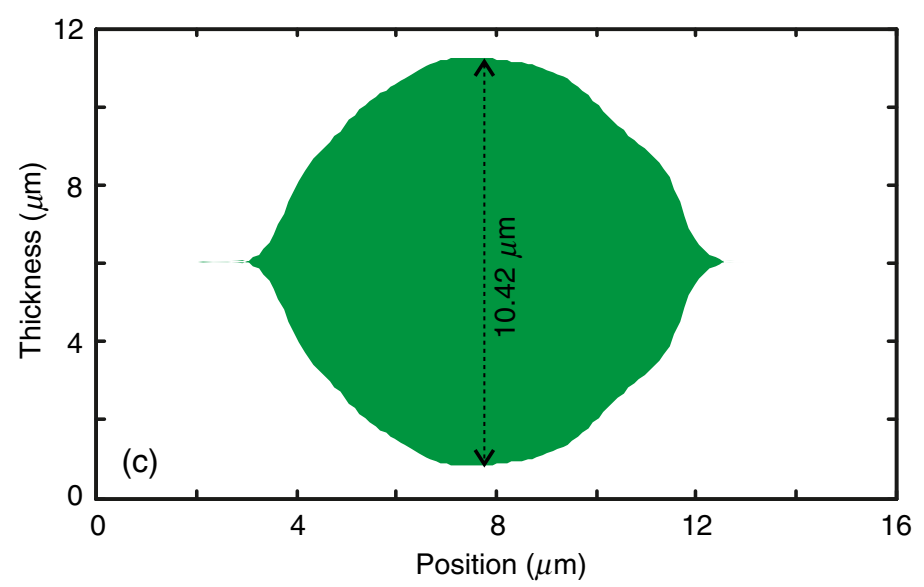

Fig. 3 Calibration of the wide field of view lateral-shearing digital holographic microscope using polystyrene microspheres of $10-\mu \mathrm{m}$ diameter: (a) continuous quantitative phase profile obtained after phase subtraction, (b) 3-D thickness distribution profile of the microsphere obtained from the phase profile, and (c) cross-sectional thickness profile of the microsphere along the line shown in (a). 


$$
\begin{aligned}
U_{\mathrm{O}}(x, y, z=d)= & \mathfrak{I}^{-1}\{\operatorname{filt}[\mathfrak{s}\{U(x, y, z=0)\}] \\
& \left.\times \exp \left[i k \sqrt{1-\lambda^{2} f_{X}^{2}-\lambda^{2} f_{Y}^{2}} d\right]\right\},
\end{aligned}
$$

where $U_{\mathrm{O}}$ is the complex amplitude distribution of the object at the image plane situated at a distance $d$ from the hologram plane and filt $[\mathfrak{S}\{U(x, y, z=0)\}]$ is the filtered angular spectrum (obtained by Fourier transforming the hologram illuminated by the digital version of the reference wavefront) and the exponential term in the free-space propagation function. An angular spectrum approach describes the wavefront propagation over short distances (no assumptions on the minimum distance of propagation-no paraxial approximation) compared with the size of the array (hologram). This is in contrast to the FresnelKirchoff integral that assumes propagation distances much larger than the hologram dimensions. Thus, in the case of DHIM, angular spectrum propagation approach is ideal for image retrieval and makes the experimental setup compact. Another advantage of this approach is that it can separate out the different diffracted components (undiffracted reference, virtual object component, and real-object component) in the frequency domain (only the frequency information about the virtual/real object is propagated), so there will not be any overlap between the three components in the reconstruction plane (image plane). ${ }^{9,13-15}$ In the case of the developed microscope, the image plane of the system was situated exactly at the detector array (hologram plane), so the propagation distance $d$ in Eq. (2) is 0 and, hence, the reconstruction just involves a Fourier analysis of the resulting fringes, without the need for propagation. ${ }^{40}$ Thus, the determination of the position of the pin-hole (from the CCD array) for the computation of the reference wavefront is not necessary. In the reconstructions, a circular filter of radius equal to 160 pixels centered at the sideband of the frequency spectrum was used to select the object frequency components. The phase was computed after inverse Fourier transforming the filtered hologram spectrum. For each set of object holograms (holograms recorded with the object in the field of view), a reference hologram (hologram recorded with only the medium surrounding the object in the field of view) is recorded for phase compensations. The reference phase is subtracted from the object phase to obtain the phase difference distribution $(\Delta \phi)$. This process nullifies most of the phase due to aberrations (which remained the same between the exposures) and brings out the phase due to the object. ${ }^{9,13-15}$ This phase difference is related to the object thickness through

$\Delta \phi(x, y)=\frac{2 \pi}{\lambda}\left(n_{\mathrm{O}}-n_{\mathrm{B}}\right) L(x, y)$,

where $n_{\mathrm{O}}$ and $n_{\mathrm{B}}$ are the constant average refractive indices of the object and the background, respectively, and $L$ is the spatially varying thickness of the object. Equation (3) can be used to determine either the thickness or the optical thickness, $\left(n_{\mathrm{O}}-n_{\mathrm{B}}\right) L$, of the cells.

It should be noted that parallel GP may introduce spherical aberrations, on a diverging beam. However, since the region of the wavefront detected is very small $(\sim 5 \mathrm{~mm} \times 5 \mathrm{~mm}$ - detector dimension) compared with the size of the wavefront incident on

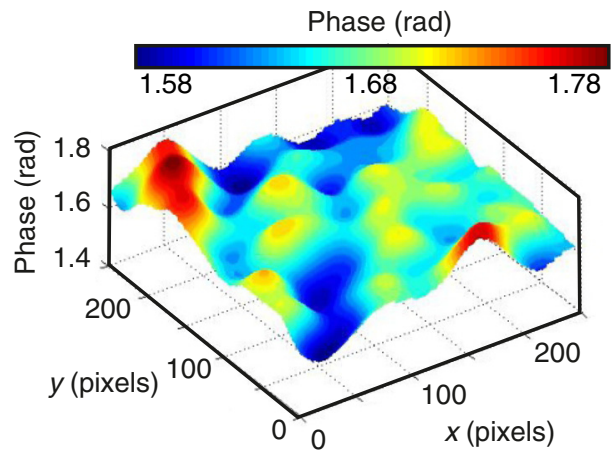

(a)

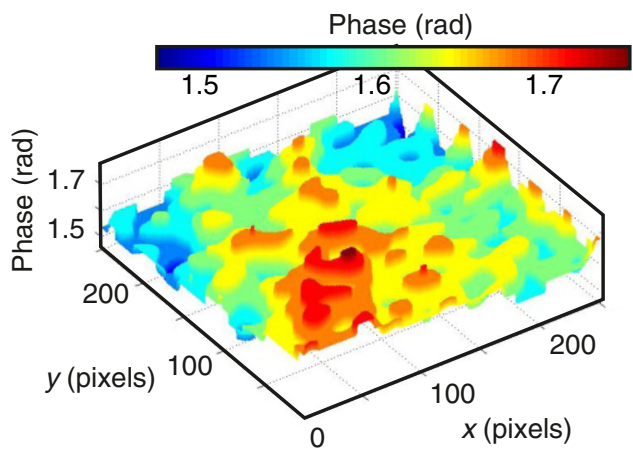

(c)

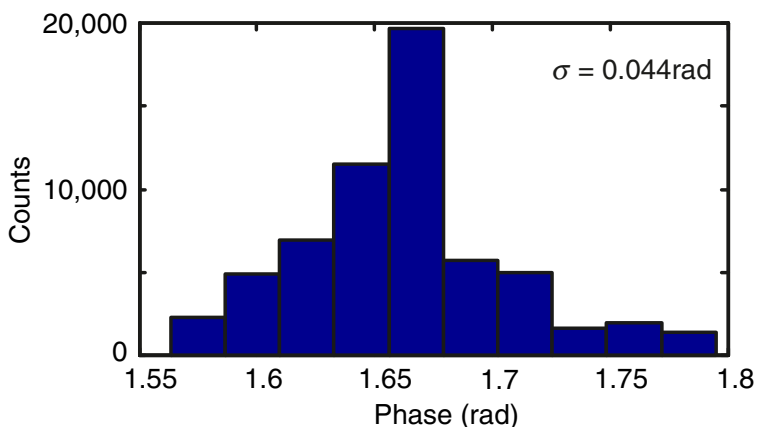

(b)

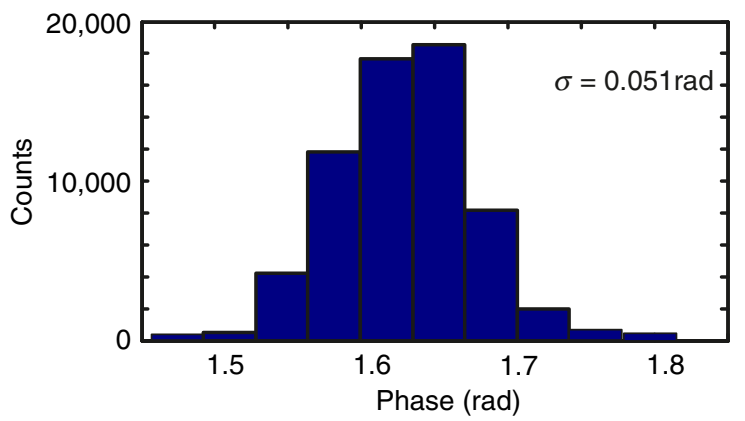

(d)

Fig. 4 Spatial stability of the wide field of view common-path digital holographic microscope. Spatial variation of phase computed from holograms recorded using a CCD array and a VGA webcam sensor are shown in (a) and (c), respectively. Histograms of the phase distributions for obtained using CCD array and webcam sensor are shown in (b) and (d), respectively. 
the sharing GP $(\sim 5 \mathrm{~cm} \times 5 \mathrm{~cm})$, the detected wavefront can be approximated as a plane wavefront and, hence, the effect of spherical aberration due to the shearing plate can be considered negligible. The advantage of digital holography is that even these aberrations can be compensated numerically. ${ }^{41,42}$

\section{Testing and Calibration of the Microscope}

System is calibrated by recording the holograms of polystyrene microspheres of diameter $10 \mu \mathrm{m} \pm 0.25 \mu \mathrm{m}$, immersed in microscope immersion oil. The refractive index of the material of the microsphere is 1.58 and that of the immersion oil is 1.52 . Figure 3(a) shows the reconstructed unwrapped phase distribution of the microsphere recorded using a CCD array. This phase distribution is plugged into Eq. (3) along with the refractive index values to obtain the thickness profile of the microsphere as shown in Figs. 3(b) and 3(c) [half of the thickness, along the line in Fig. 3(a) is used to construct the top and bottom portions]. The thickness measurement was carried out for several microspheres, which provided a mean thickness value of $10.11 \mu \mathrm{m} \pm 0.19 \mu \mathrm{m}$, which is very close to the manufacturer specified value of $10 \mu \mathrm{m}$. The thickness measurement of these microspheres validates the 3-D imaging capability of the developed DHIM.

A low-cost version of the microscope is also developed using a VGA $(640 \times 480$ pixels) webcam sensor (color, pixel pitch $3.2 \mu \mathrm{m}$ ) to record holograms. Calibration of the low-cost system is also done the same way as in the case of the system with a CCD array. The spatial phase stability value of the microscope provides its limit on imaging axial thickness variations. It is computed from the spatial phase variation obtained with a blank microscope slide. Figure 4(a) shows the spatially varying phase distribution obtained with a CCD array as the recording device. A histogram of the spatial phase variation is shown in Fig. 4(b), and the standard deviation of this phase distribution is its spatial phase stability. The mean of the spatial phase stability computed from several holograms serves as the spatial stability value of the microscope and determines its axial resolution. Spatial stability computations for a webcam sensor as the recording device are shown in Figs. 4(c) and 4(d). The mean of the obtained spatial stabilities was $3.62 \mathrm{~nm}$ for the setup using a CCD array and $4.15 \mathrm{~nm}$ for setup using a webcam array. The spatial phase variation was also computed without the microscope slide and was found to be 1.62 and $1.91 \mathrm{~nm}$ for the setups using a CCD array and a webcam array, respectively. The accuracy of the reconstructed phase depends on the amount of unwanted spatial variation in the reconstructed phase. In the described setup, since GPs are used for shearing the beam as well as for holding the pin-hole, the accuracy of the reconstructed phase decreases, due to the phase variations occurring due to the GPs, compared with the other two-beam setups where subnanometer spatial phase stability (accuracy) has been achieved. $^{24}$

One of the advantages of the common-path DHIM is that it can provide subnanometer temporal stability which is required
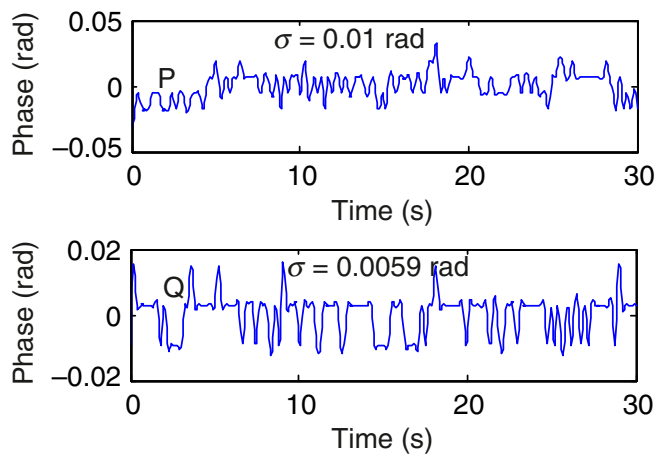

(a)
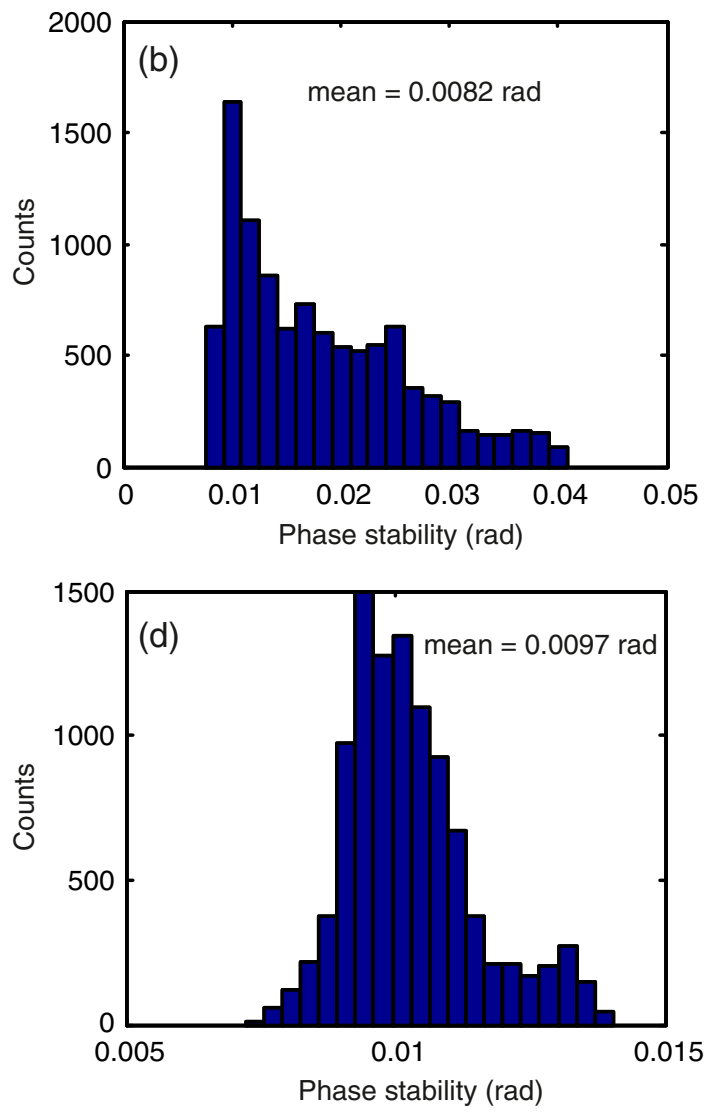

(c)

Fig. 5 Temporal phase stability of the wide field of view common-path digital holographic microscope using (a) and (b) CCD array and (c) and (d) VGA webcam sensor. (a) and (c) shows the time evolution of phase at two spatial points, and (b) and (d) is the histogram of the temporal phase variation for CCD array and webcam, respectively. 
to study cell membrane fluctuations. ${ }^{19-25,28,35-37}$ Consequently, the measurement of temporal stability of the developed common-path system is necessary before its use in dynamic imaging of cells. For measurement of the temporal phase stability, a time series of holograms with a blank microscope slide (object holograms) in the sample holder is recorded. The mean of the standard deviations of the time variation of the phase (obtained from hologram recorded at each time instance) computed at several spatial points provides the temporal phase stability. ${ }^{23-25,28,35-37}$ This is repeated by recording several time series, and the average of these phase stabilities is used as the value of the temporal phase stability of the system. For the CCD array, holograms were recorded at the rate of $15 \mathrm{~Hz}$ for a total period of $30 \mathrm{~s}$, and, in the case of the webcam sensor, holograms were recorded at $25 \mathrm{~Hz}$ for $30 \mathrm{~s}$ to compute the temporal stability. Figure 5 shows the temporal stability measurement of the developed system. The histograms shown in Figs. 5(b) and 5(d) are the distribution of the standard deviation of phase variations due to mechanical vibrations for each pixel of the reconstructed image. From Fig. 5, it can be seen that the developed device provides subnanometer temporal stabilities ( $0.81 \mathrm{~nm}$ for setup using CCD and $0.94 \mathrm{~nm}$ for setup using webcam over a period of $30 \mathrm{~s}$ ), owing to its common-path nature. Temporal stabilities were also computed without the microscope slide and were found to be 0.45 and $0.57 \mathrm{~nm}$ over a period of
$30 \mathrm{~s}$ for the setups employing the CCD array and webcam array, respectively.

Sampling of one of the beams using a pin-hole to create a separated reference beam leads to higher field of view $(150 \mu \mathrm{m} \times 110 \mu \mathrm{m})$ and higher spatial stability $(1.62 \mathrm{~nm})$ compared with the self-referencing version with field of view of $90 \mu \mathrm{m} \times 110 \mu \mathrm{m}$ and spatial stability of above $2 \mathrm{~nm}$. The common-path nature of the microscope provides subnanometer temporal stability also. The field of view in the case of the present setup depends on two parameters: (i) amount of magnification and (ii) size of the imaging array. When compared with the lateral-shearing self-referencing digital holographic microscope, ${ }^{32}$ where only half of the detector array can be used for recording information, the present setup offers double the field of view, while remaining essentially a common-path technique. This field of view is the same as the one offered by Mach-Zehnder interferometer based two-beam digital holographic microscopes. The field of view can be increased by increasing the recording array size.

\section{Imaging of Red Blood Cells Using Wide Field of View Microscope}

After calibrating the system and determining its spatial and temporal stabilities, it is used for 3-D imaging of red blood cells and their parameter extraction. For this thin blood smears obtained
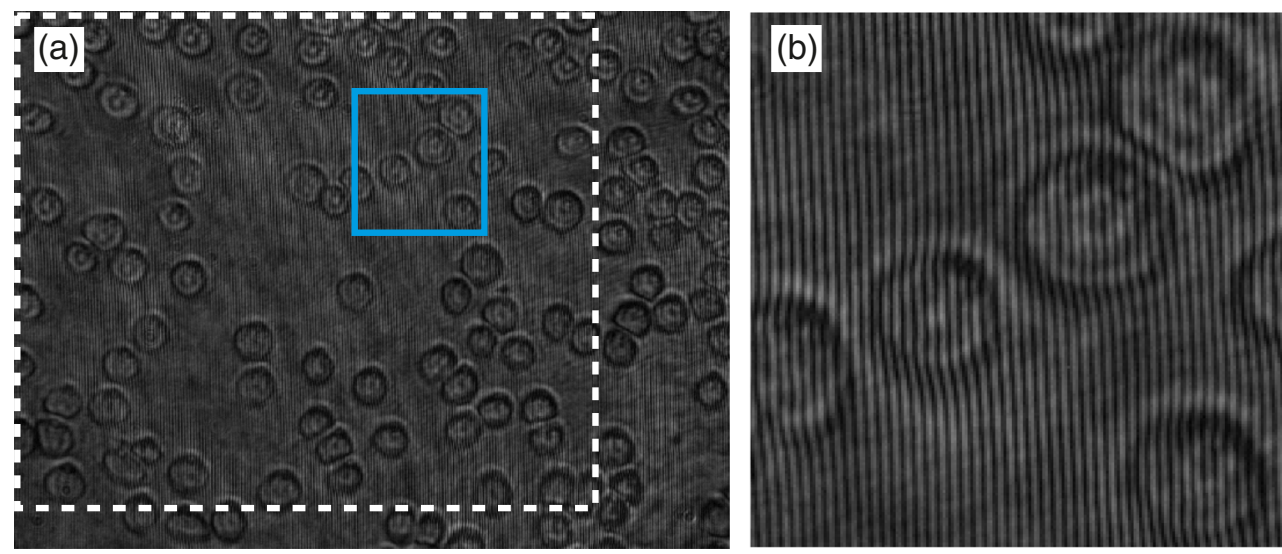

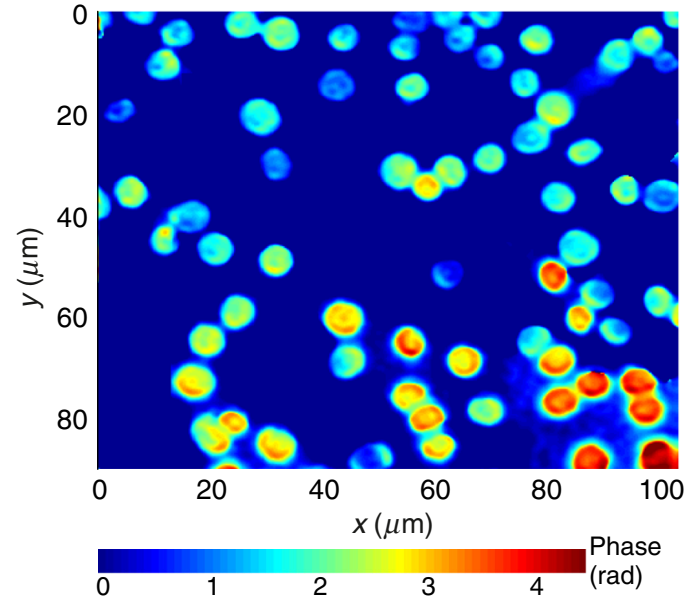

(c)
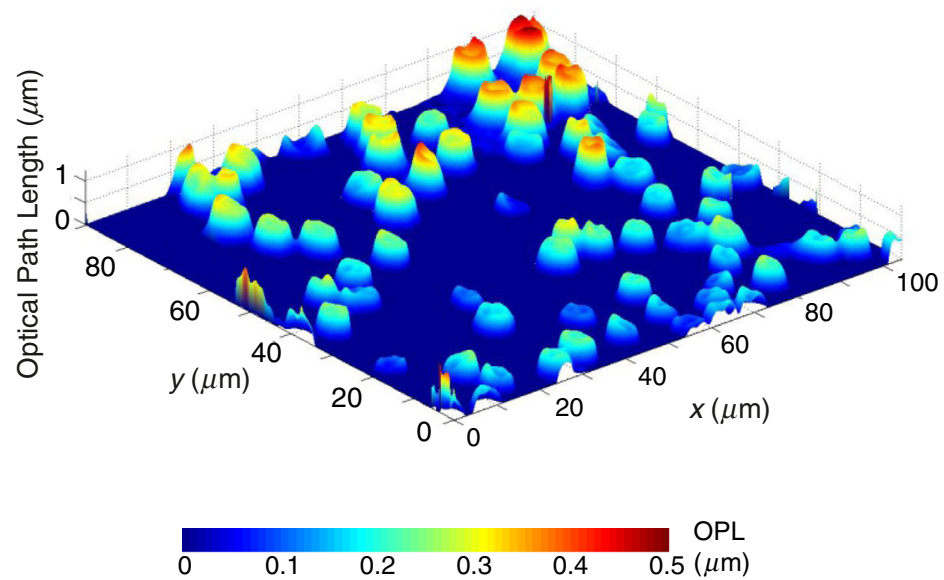

(d)

Fig. 6 (a) Hologram recorded with CCD array, (b) zoomed region inside the blue rectangle, (c) quantitative phase distribution inside the white (dashed) rectangle, and (d) optical thickness distribution computed from the quantitative phase information. 
from healthy donors were made on microscope glass slides and images taken using the developed microscope. As mentioned, the biggest advantage of this microscope is that its full field of view can be utilized, unlike the one employing self-referencing lateral-shearing geometry. Figure 6(a) shows the hologram of red blood cell distribution (obtained from a healthy individual) recorded using an 8-bit CCD array of pixel pitch $4.65 \mu \mathrm{m}$. The portion inside the blue rectangle [in Fig. 6(a)] is shown in Fig. 6(b), which clearly shows the interference fringes and their modulation. The quantitative phase contrast image obtained after phase subtraction is shown in Fig. 6(c). The optical thickness distribution computed by plugging this phase distribution into Eq. (3) provides the 3-D profile of the cells shown in Fig. 6(d).

The low-cost version of the microscope has a reduced field of view since the sensor area is smaller compared with that of CCD array. However, the cost of the webcam sensor is only a fraction of that of a CCD array, making the microscope using webcam cost $\sim 100$ times less than the one using CCD array. Figure 7(a) shows the hologram recorded using the webcam array. The region of interest inside the black rectangle is shown in Fig. 7(b), which shows the recorded interference fringes. The obtained quantitative phase profile of the cells after phase subtraction is shown in Fig. 7(c), and the optical thickness distribution is shown in Fig. 7(d).

The time variation in the thickness profile provides the dynamic parameters related to the cells. For the CCD array, holograms were recorded (field of view $1024 \times 768$ pixels $150 \mu \mathrm{m} \times 110 \mu \mathrm{m})$ at the rate of $15 \mathrm{~Hz}$ for $30 \mathrm{~s}$. The reconstructed phase profile is used to compute the spatially varying thickness profile of the cell at each time instance. Figure 8(a) shows the reconstructed phase profile of the cells in the field of view at one time instance. Each cell is identified by thresholding the phase distribution by the average of the phase value of the background (region where there were no cells) and marked. Inset of the figure shows the 3-D rendering of the optical thickness distribution of the cell marked inside the rectangle. Fluctuation profile of the cell is obtained by the use of the standard deviation of the time-varying thickness profile and is shown in Fig. 8(b). Inset of this figure shows the 3-D rendering of the fluctuation profile of the cell inside the rectangle. Optical cell volume at each time instance is the sum of the thickness at the pixel multiplied by the area occupied by each pixel, which in turn depends on the lateral magnification of the system. Figure 8 (c) shows the time variation in optical volume of different cells marked in Fig. 8(a). Fourier analysis of the time-varying thickness profile provides information about the frequency of thickness fluctuations at different points inside the cell as shown in Fig. 8(d). Here also the inset shows the 3-D rendering of the frequency distribution for the cell inside the rectangle. Measured red blood cell parameters (obtained from 65 cells) using CCD array to record holograms are given in Table 1.

Healthy red blood cell distributions are imaged using the microscope employing a VGA webcam array. The field of view in the case of webcam sensor is $\sim 50 \mu \mathrm{m} \times 65 \mu \mathrm{m}$. Here, holograms for $30 \mathrm{~s}$ were recorded but at the rate of $30 \mathrm{~Hz}$. Figure 9(a) shows the optical thickness distribution of the healthy red blood cells imaged using the webcam sensor
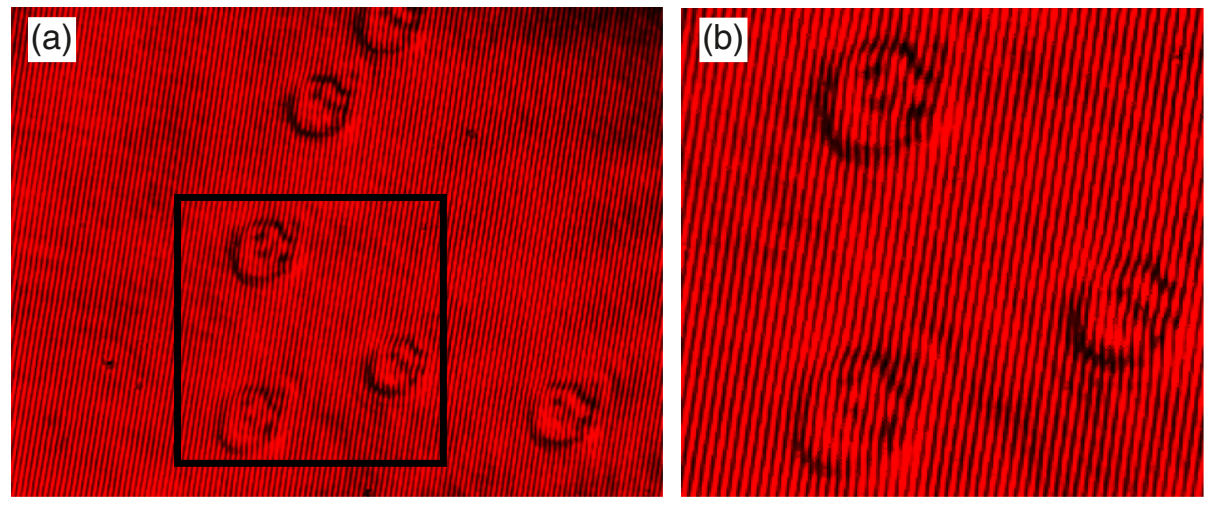

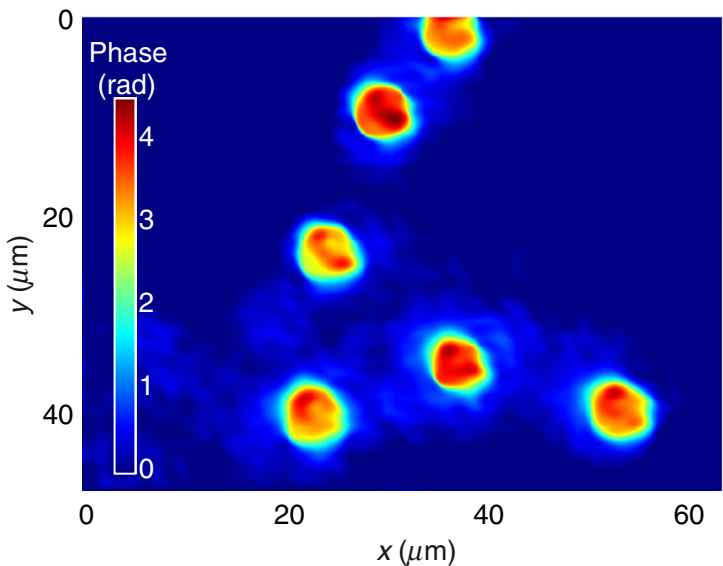

(c)

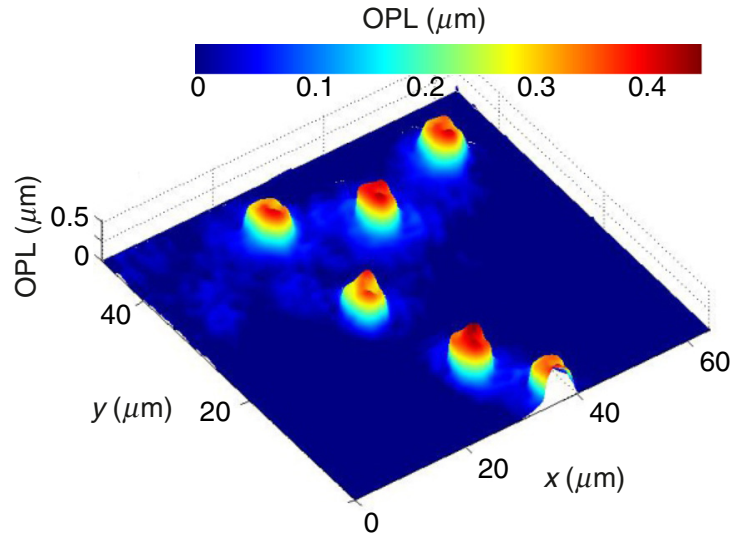

(d)

Fig. 7 Results obtained with webcam array as the recording device: (a) hologram recorded with webcam array, (b) zoomed region inside the black rectangle, (c) quantitative phase distribution for the hologram shown in (a), and (d) optical thickness distribution computed from the quantitative phase information. 


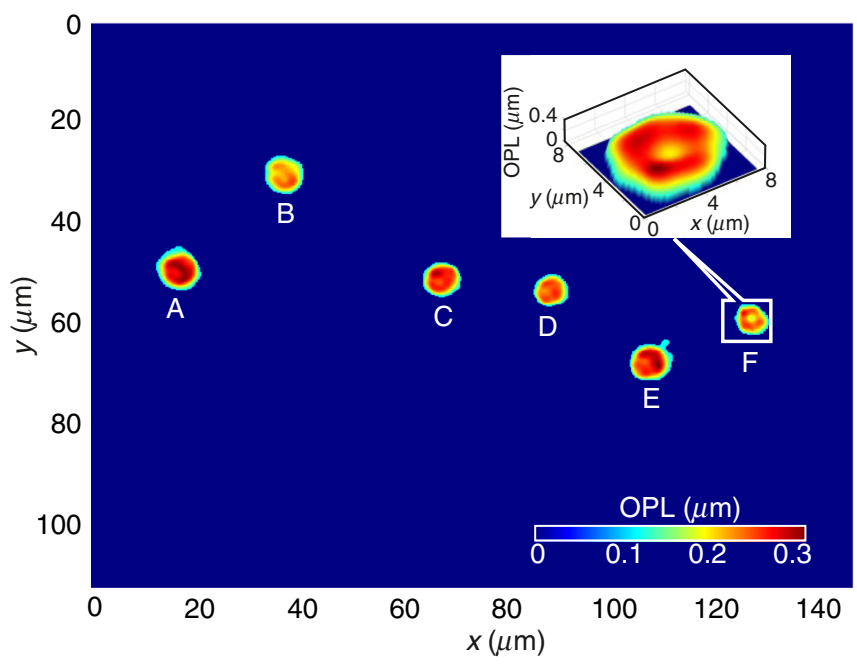

(a)
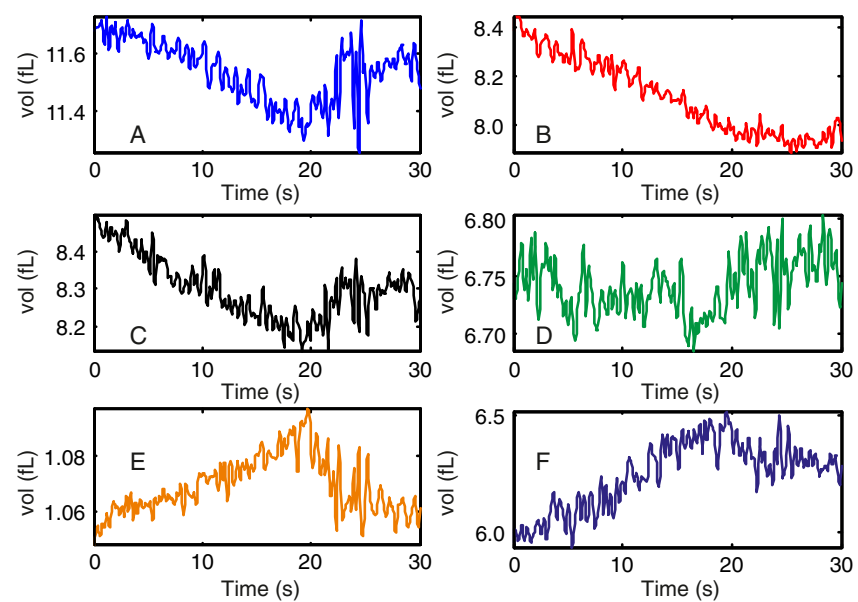

(c)

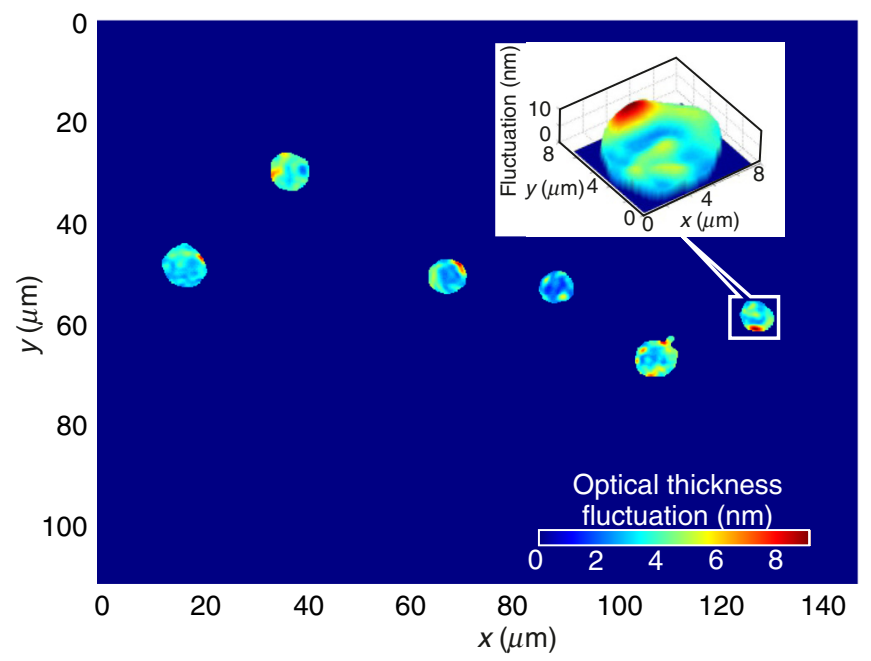

(b)

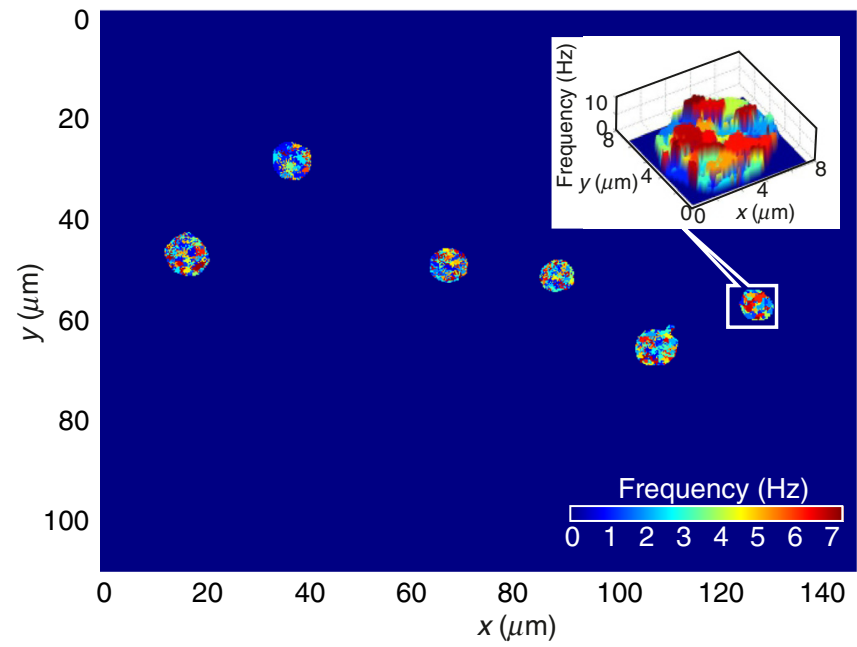

(d)

Fig. 8 Imaging of cell dynamics using CCD array. (a) Optical path length distribution of the cells in the field of view reconstructed from the phase profile. Inset shows the 3-D optical thickness profile of the cell inside the region of interest marked by the white rectangle. (b) Optical thickness fluctuation at each point on the cell along with the 3-D rendering of the optical fluctuation profile for the cell inside the white rectangle. (c) Time variation of optical volume [for the cells marked in (a)]. (d) Frequency of thickness oscillations at each point on the cell (inset shows the 3-D rendering of the frequency profile for the cell inside the white rectangle).

Table 1 Static and dynamic parameters of healthy red blood cells measured using wide field of view lateral-shearing digital holographic microscope.

\begin{tabular}{llcc}
\hline & & \multicolumn{2}{c}{ Measured value $($ mean $\pm \mathrm{std})$} \\
\cline { 3 - 4 } $\begin{array}{l}\text { Sr. } \\
\text { No. }\end{array}$ & Parameter & $\begin{array}{c}\text { Using CCD } \\
\text { array }\end{array}$ & Using webcam \\
\hline 1 & Diameter $(\mu \mathrm{m})$ & $7.41 \pm 0.54$ & $7.56 \pm 0.35$ \\
2 & $\begin{array}{l}\text { Mean value of optical } \\
\text { thickness }(\mu \mathrm{m})\end{array}$ & $0.288 \pm 0.061$ & $0.259 \pm 0.022$ \\
3 & $\begin{array}{l}\text { Coefficient of variation of } \\
\text { optical thickness }\end{array}$ & $0.2132 \pm 0.0323$ & $0.2442 \pm 0.0287$ \\
& & &
\end{tabular}

Table 1 (Continued).

\begin{tabular}{llcr}
\hline & & \multicolumn{2}{c}{ Measured value (mean $\pm \mathrm{std})$} \\
\cline { 3 - 4 } $\begin{array}{l}\text { Sr. } \\
\text { No. }\end{array}$ & \multicolumn{1}{c}{ Parameter } & $\begin{array}{c}\text { Using CCD } \\
\text { array }\end{array}$ & Using webcam \\
\hline 4 & Projected area $\left(\mu \mathrm{m}^{2}\right)$ & $43.19 \pm 9.41$ & $41.43 \pm 5.82$ \\
5 & Optical volume (fL) & $7.64 \pm 1.86$ & $8.12 \pm 1.06$ \\
6 & $\begin{array}{l}\text { Optical total surface } \\
\text { area }\left(\mu \mathrm{m}^{2}\right)\end{array}$ & $88.86 \pm 15.67$ & $84.51 \pm 9.27$ \\
7 & $\begin{array}{l}\text { Surface area/optical } \\
\text { volume ratio } \times 10^{-6}\left(\mathrm{~m}^{-1}\right)\end{array}$ & $11.22 \pm 0.56$ & $10.85 \pm 0.78$ \\
& & & \\
\hline
\end{tabular}


Table 1 (Continued)

\begin{tabular}{llcr} 
& & \multicolumn{2}{c}{ Measured value (mean \pm std) } \\
\cline { 3 - 4 } $\begin{array}{l}\text { Sr. } \\
\text { No. }\end{array}$ & \multicolumn{1}{c}{ Parameter } & $\begin{array}{c}\text { Using CCD } \\
\text { array }\end{array}$ & Using webcam \\
\hline 8 & Sphericity & $0.245 \pm 0.015$ & $0.258 \pm 0.017$ \\
9 & $\begin{array}{l}\text { Amplitude of thickness } \\
\text { fluctuation }(\mathrm{nm})\end{array}$ & $3.23 \pm 1.03$ & $3.11 \pm 1.13$ \\
10 & Volume fluctuation (fL) & $0.106 \pm 0.005$ & $0.091 \pm 0.006$ \\
11 & $\begin{array}{l}\text { Mean peak frequency of } \\
\text { thickness fluctuation }(\mathrm{Hz})\end{array}$ & $3.37 \pm 0.91$ & $3.93 \pm 0.92$ \\
\hline
\end{tabular}

along with the 3-D visualization of one of the cells (inside the white rectangle). The reconstructed time-evolving thickness variation provides the optical thickness fluctuation profile as shown in Fig. 9(b), which also shows the 3-D thickness

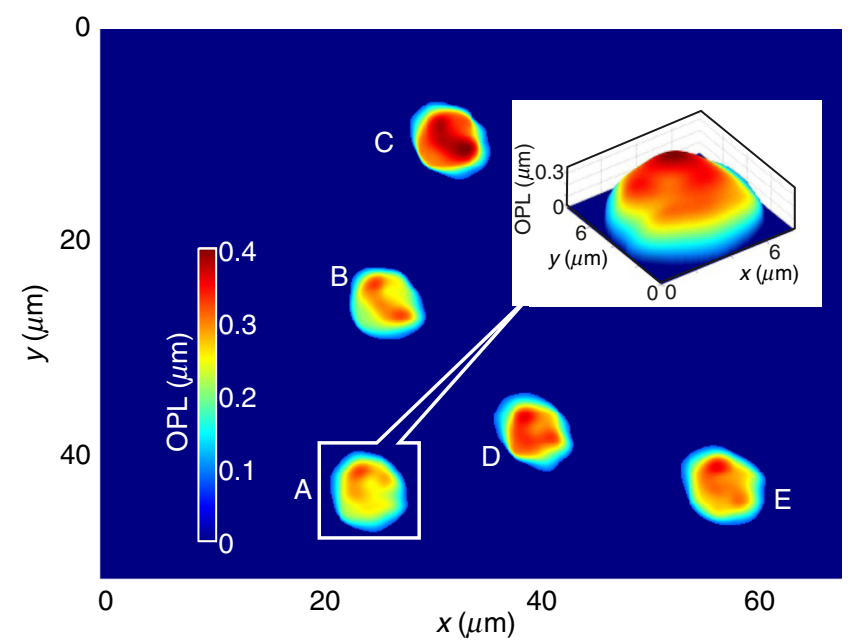

(a)
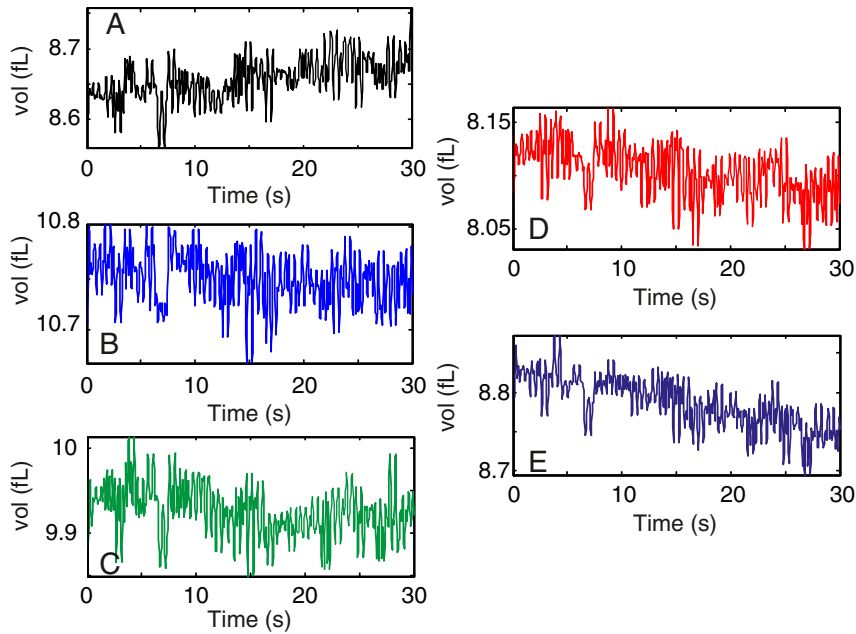

(c) fluctuation profile for the marked cell. The time-evolving volume for the cells marked (A to E) in Fig. 9(a) is shown in Fig. 9(c). The frequency profile of the cells [Fig. 9(d)] obtained by Fourier analysis of the time-evolving thickness profile at each point on the cell is used to calculate the frequency of optical thickness oscillations of the cells. Table 1 shows the measured blood cell parameters for the holograms recorded by the webcam array (for 52 cells).

\section{Conclusions}

An easy to implement, compact, and wide field of view lateralshearing digital holographic microscope was developed using a laser diode module, a shearing plate, a focusing lens, and a digital array. A commercially available fused silica GP was used for shearing and duplicating the incident object wavefront. The reflectivity of this GP is $\sim 4 \%$ (for both faces). Since most of the light is transmitted, the intensity of the light reflected from the front and back surfaces is almost equal. This will lead to a high fringe contrast ratio as the beam intensities are same. By contrast, the pin-hole reduces the intensity of the reference

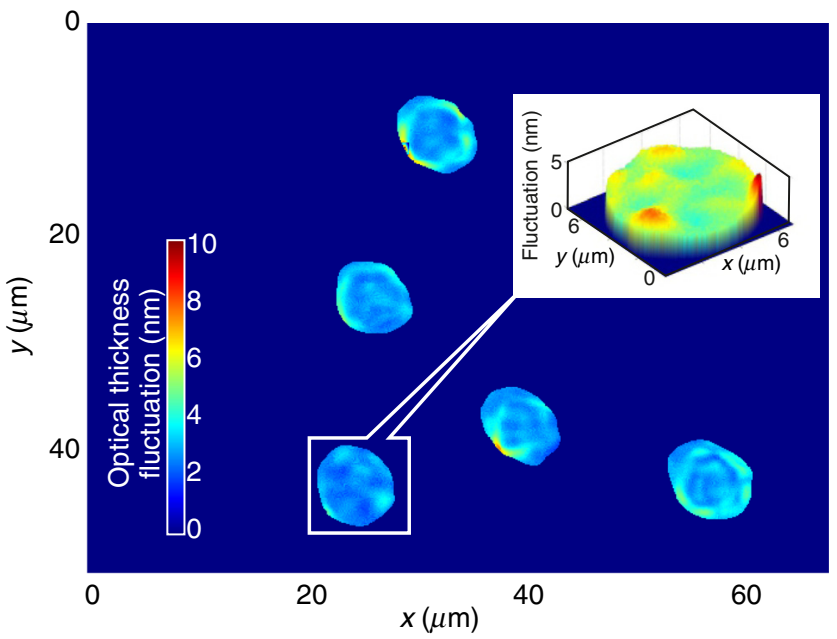

(b)

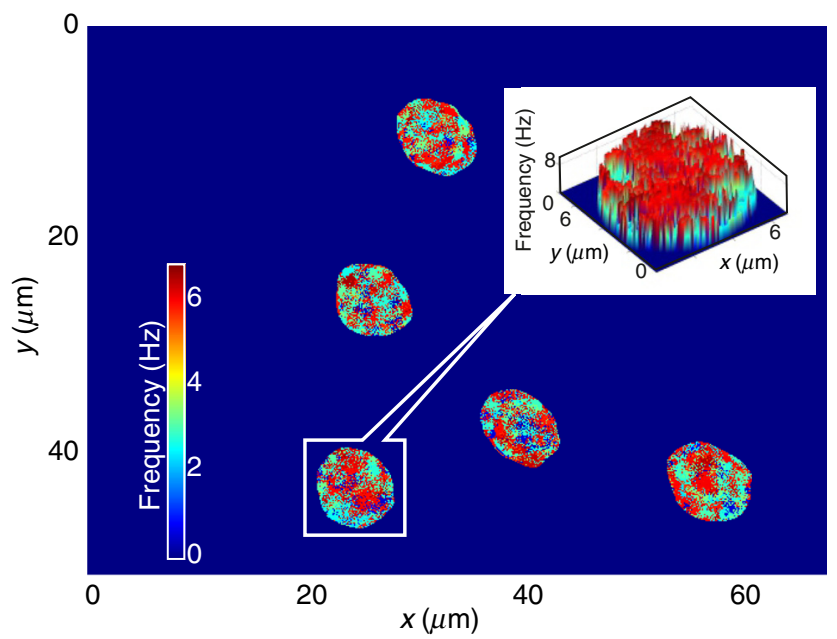

(d)

Fig. 9 Imaging of cell dynamics using webcam array. (a) Optical thickness distribution of the cells in the field of view obtained from the phase profile along with the 3-D optical thickness profile of the cell inside the region of interest marked by the white rectangle. (b) Optical thickness fluctuation profile of the cells along with the 3-D rendering of the optical fluctuation profile. (c) Optical volume as a function of time [for the cells marked in (a)]. (d) Change in frequency of thickness fluctuation with position inside the cells. 
beam in comparison with the object beam, which is not altered at all. The measured intensity ratio (reference to object) was $\sim 0.17$, leading to a fringe contrast of $\sim 0.7$. This fringe contrast was good enough to extract the object phase information, which can be seen from the results. The fringe contrast may be improved by placing a neutral density filter in the path of the object beam at the pin-hole plane, but this will increase the complexity of the setup. The spatial resolution of the system is determined by the numerical aperture of the imaging lens. However, in numerical reconstructions using angular spectrum propagation integral, masks are used for selecting the frequency components corresponding to the object. This may lead to reduction of spatial frequency. In the case of the developed microscope, a circular filter with a radius of 160 pixels was used. This results in the reconstructed spatial resolution of $\sim 0.9 \mu \mathrm{m}$, which is lower than the optical resolution provided by the MO lens $(\sim 0.5 \mu \mathrm{m})$. Use of a larger sized circular filter will introduce the DC (average) term in the reconstructed image, which is undesirable. A larger amount of shear (by the use of a thicker shearing plate) can increase the interference angle between the object and the reference beam, leading to higher fringe density and hence the use of circular filters with a larger size. However, larger shearing may also result in a lower overlap area between the object and the reference beams. The quality of the phase images compared with two-beam geometry is less. This may be mainly due to the use of two GPs (one for shearing and one for the pin-hole). This leads to unwanted spatial variations. Also, the use of coherent laser sources leads to the generation of laser speckles, again leading to lower quality of reconstructed phases, especially for dense object distributions. The noise can be numerically reduced by the use of nonlinear masks that truncate selected frequencies. The noise could also be reduced by the use of low temporally coherent sources, leading to reduced speckles. However, use of such sources requires a redesigning of the setup so that the path length difference between the object and the reference beams remains below the coherence length.

The main advantage of the developed microscope over the self-referencing lateral-shearing version is that its whole field of view is usable (no redundant object information). In comparison with the two-beam Mach-Zehnder-based microscope, the described technique is more temporally stable, providing subnanometer temporal resolution. This subnanometer stability makes it possible to study cell thickness fluctuations accurately; thus, the optical thickness profile of object along with the time evolution of the thickness profile can be measured accurately using this microscope. Webcam arrays are a cost-effective alternative to CCD arrays to record holograms. A low-cost version of the device was also developed using the webcam array to record holograms. The field of view in the case of VGA webcam array is less than that of the CCD array, but this can be improved using megapixel webcam arrays and reducing the magnification of the system. As the developed system is compact, it could be used for point-of-care data acquisition and cell parameter extraction, leading to label-free disease diagnosis. Presently, work is progressing in this direction.

\section{Disclosures}

The authors have no relevant financial interests in this article and no potential conflicts of interest to disclose.

\section{Acknowledgments}

This work was supported by research grant from Department of Atomic Energy-Board of Research in Nuclear Sciences (No. 2013/34/11/BRNS/504), Government of India. Arun Anand, Vani Chhaniwal, and Ali-Reza Moradi would like to thank the International Center for Theoretical Physics, Trieste, Italy, for associate fellowships.

\section{References}

1. P. Marquet et al., "Digital holographic microscopy: a noninvasive contrast imaging technique allowing quantitative visualization of living cells with subwavelength axial accuracy," Opt. Lett. 30, 468-470 (2005).

2. F. Dubois et al., "Digital holographic microscopy for the three-dimensional dynamic analysis of in vitro cancer cell migration," J. Biomed. Opt. 11, 054032 (2006).

3. B. Kember and G. von Bally, "Digital holographic microscopy for live cell applications and technical inspection," Appl. Opt. 47, A52-A61 (2008).

4. B. Rappaz et al., "Comparative study of human erythrocytes by digital holographic microscopy, confocal microscopy," Cytometry 73, 895-903 (2008).

5. B. Rappaz et al., "Spatial analysis of erythrocyte membrane fluctuations by digital holographic microscopy," Blood Cells Mol. Dis. 42, 228-232 (2009).

6. B. Rappaz et al., "Noninvasive characterization of the fission yeast cell cycle by monitoring dry mass with digital holographic microscopy," J. Biomed. Opt. 14, 034049 (2009).

7. B. Kemper et al., "Label-free quantitative cell division monitoring of endothelial cells by digital holographic microscopy," J. Biomed. Opt. 15, 036009 (2010).

8. D. Shin et al., "Optofluidic system for three-dimensional sensing and identification of micro-organisms with digital holographic microscopy," Opt. Lett. 35, 4066-4068 (2010).

9. A. Anand, V. K. Chhaniwal, and B. Javidi, "Imaging embryonic stem cell dynamics using quantitative 3D digital holographic microscopy," IEEE Photonics J. 3, 546-554 (2011).

10. P. Ferraro, A. Wax, and Z. Zalevsky, Coherent Light Microscopy: Imaging and Quantitative Phase Analysis, Springer, Berlin (2011).

11. P. Memmolo et al., "Identification of bovine sperm head for morphometry analysis in quantitative phase-contrast holographic microscopy," Opt. Express 19, 23215-23226 (2011).

12. I. Moon et al., "Cell identification with computational 3-D holographic microscopy," Opt. Photonics News 22(6), 18-23 (2011).

13. A. Anand et al., "Automatic identification of malaria infected RBC with digital holographic microscopy using correlation algorithms," IEEE Photonics J. 4, 1456-1464 (2012).

14. A. Anand and B. Javidi, "Digital holographic microscopy for automated 3D cell identification: an overview," Chin. Opt. Lett. 12, 060012 (2014).

15. A. Anand, I. Moon, and B. Javidi, "Automated disease identification with 3D optical imaging: a medical diagnostic tool," Proc. IEEE 105, 924-946 (2017).

16. T. Kreis, Handbook of Holographic Interferometry: Optical and Digital Methods, Wiley-VCH, Weinheim (2005).

17. U. Schnars and W. Jueptner, Digital Holography: Digital Hologram Recording, Numerical Reconstruction and Related Techniques, Springer, Berlin (2005).

18. U. Schnars and W. Jueptner, "Digital recording and numerical reconstruction of holograms," Meas. Sci. Technol. 13, R85-R101 (2002).

19. G. Popescu et al., "Erythrocyte structure and dynamics quantified by Hilbert phase microscopy," J. Biomed. Opt. 10, 060503 (2005).

20. G. Popescu et al., "Observation of dynamic subdomains in red blood cells,” J. Biomed. Opt. 11, 040503 (2006).

21. M. Mir, K. Tangella, and G. Popescu, "Blood testing at the single cell level using quantitative phase and amplitude microscopy," Biomed. Opt. Express 2, 3259 (2011).

22. N. T. Shaked et al., "Quantitative microscopy and nanoscopy of sickle red blood cells performed by wide field digital interferometry," J. Biomed. Opt. 16, 030506 (2011). 
23. N. T. Shaked, "Quantitative phase microscopy of biological samples using a portable interferometer," Opt. Lett. 37, 2016-2018 (2012).

24. B. Bhaduri et al., "Diffraction phase microscopy with white light," Opt. Lett. 37, 1094-1096 (2012).

25. A. Anand et al., "Single beam Fourier transform digital holographic quantitative phase microscopy," Appl. Phys. Lett. 104, 103705 (2014).

26. V. Mico et al., "Spatially-multiplexed interferometric microscopy (SMIM): converting a standard microscope into a holographic one," Opt. Express 22, 14929-14943 (2014).

27. Y. Kim et al., "Profiling individual human red blood cells using common-path diffraction optical tomography," Sci. Rep. 4, 6659 (2014).

28. S. Mahajan et al., "Highly stable digital holographic microscope using Sagnac interferometer," Opt. Lett. 40, 3743-3746 (2015).

29. V. Bianco et al., "Endowing a plain fluidic chip with micro-optics: a holographic microscope slide," Light Sci. Appl. 6, e17055 (2017).

30. P. Bon et al., "Quadriwave lateral shearing interferometry for quantitative phase microscopy of living cells," Opt. Express 17, 13080-13094 (2009).

31. G. Coppola et al., "Digital self-referencing quantitative phase microscopy by wavefront folding in holographic image reconstruction," Opt. Lett. 35, 3390-3392 (2010).

32. J. Jang et al., "Self-reference quantitative phase microscopy for microfluidic devices," Opt. Lett. 35, 514-516 (2010).

33. B. Kemper et al., "Simplified approach for quantitative digital holographic phase contrast imaging of living cells," J. Biomed. Opt. 16, 026014 (2011).

34. J. Jang et al., "Self-reference quantitative phase microscopy for microfluidic devices," Opt. Lett. 35, 514-516 (2010).

35. A. S. G. Singh et al., "Lateral-shearing digital holographic imaging of small biological specimens," Opt. Express 20, 23617-23622 (2012).

36. A. Anand et al., "Compact, common path quantitative phase microscopic techniques for imaging cell dynamics," Pramana 82, 71-78 (2014).

37. V. K. Chhaniwal et al., "Quantitative phase contrast imaging with compact DHM employing Lloyd's mirror," Opt. Lett. 37, 5127-5129 (2012).

38. A. Calabuig et al., "Common-path configuration in total internal reflection digital holography microscopy," Opt. Lett. 39, 2471-2474 (2014).

39. J. W. Goodman, Introduction to Fourier Optics, McGraw-Hill, New York (1996).

40. M. Takeda, H. Ina, and S. Kobayashi, "Fourier-transform based fringe pattern analysis for computer based topography and interferometry," J. Opt. Soc. Am. 72, 156-160 (1982).

41. P. Ferraro et al., "Compensation of the inherent wave front curvature in digital holographic coherent microscopy for quantitative phase-contrast imaging," Appl. Opt. 42, 1938-1946 (2003).

42. L. Miccio et al., "Direct full compensation of the aberrations in quantitative phase microscopy of thin objects by a single digital hologram," Appl. Phys. Lett. 90, 041104 (2007).

Priyanka Vora received her $\mathrm{PhD}$ in the area of 3D microscopy and its applications in 2017 from the M. S. University of Baroda. Her areas of research include application of 3D microscopy in quantification of plant and animal cells.

Vismay Trivedi received his master's degree in applied physics from the M. S. University of Baroda in 2012. Presently he is pursuing his $\mathrm{PhD}$ in the area of laser speckles. His research interests include laser speckle based measurements, 3D imaging and microcopy.

Swapnil Mahajan received his master's degree in applied physics from the M. S. University of Baroda in 2012. Presently he is pursuing his $\mathrm{PhD}$ in the area of $3 \mathrm{D}$ microscopy and its applications. His research interests include 3D interference microscopy and phase retrieval.

Nimit Patel received his master's degree in applied physics from the M. S. University of Baroda in 2010. Presently he is pursuing his PhD in the area of design and fabrication of compact microscopes. His research interests include design of hand-held 3D microscopes and its applications in cell imaging.

Mugdha Joglekar received her master's degree in applied physics from the M. S. University of Baroda in 2016. Presently she is pursuing her PhD in the area of optical coherence tomography.

Vani Chhaniwal received her PhD from the M. S. University of Baroda in 2005 in the area of optical interferometry and its applications in measurement and testing. She was a BOYSCAST postdoc fellow at University of Stuttgart. She was awarded ICTP regular associateship in 2012. Her research areas include interferometry for microscopy, laser speckles and optical instrumentation.

Ali-Reza Moradi received his PhD in optics from the Institute for Advanced Studies in Basic Sciences (IASBS) in 2009. He is presently with the Optics Research Center of IASBS and the Institute for Research in Fundamental Sciences. He has more than 45 publications in peer-reviewed journals and conference proceedings. He was awarded the ICTP junior associate fellowship in 2012 and the ICTP TRIL award in 2007. Research areas include 3D microscopy and optical micromanipulation and their applications in soft matter.

Bahram Javidi is a board of trustees distinguished professor at the University of Connecticut. He is the author of more than 700 articles in peer-reviewed journals and conference proceedings. He is a fellow of many International Scientific Societies. He is also a recipient of many prestigious awards including Humboldt price, SPIE award for technology achievement etc. His research areas include 3D sensing and imaging, 3D visualization and display, 3D image processing, recognition and tracking as well as digital holography.

Arun Anand received his $\mathrm{PhD}$ in applied physics from the M. S. University of Baroda in 2003 . He is presently a faculty member at the same university. He has more than 100 publications in peerreviewed journals and conference proceedings. He was awarded the ICTP regular associate fellowship in 2014. Research areas include digital holographic microscopy, 3D cell imaging and disease identification, fringe projection and optical instrumentation. 